\title{
What an Entangled Web We Weave: An Information-Centric Approach to Time-Evolving Socio-Technical Systems
}

\author{
Markus Luczak-Roesch • Kieron \\ O'Hara • Jesse David Dinneen • Ramine \\ Tinati
}

the date of receipt and acceptance should be inserted later

\begin{abstract}
A new layer of complexity, constituted of networks of information token recurrence, has been identified in socio-technical systems such as the Wikipedia online community and the Zooniverse citizen science platform. The identification of this complexity reveals that our current understanding of the actual structure of those systems, and consequently the structure of the entire World Wide Web, is incomplete, which raises novel questions for data science research but also from the perspective of social epistemology. Here we establish the principled foundations and practical advantages of analyzing information diffusion within and across Web systems with Transcendental Information Cascades, and outline resulting directions for future study in the area of socio-technical systems. We also suggest that Transcendental Information Cascades may be applicable to any kind of time-evolving system that can be observed using digital technologies, and that the structures found in such systems comprise properties common to all naturally occurring complex systems.
\end{abstract}

\author{
M. Luczak-Roesch \\ Victoria University of Wellington \\ School of Information Management \\ E-mail: markus.luczak-roesch@vuw.ac.nz \\ K. O'Hara \\ University of Southampton \\ Web and Internet Science Group \\ E-mail: kmo@ecs.soton.ac.uk \\ J. D. Dinneen \\ Victoria University of Wellington \\ School of Information Management \\ E-mail: jesse.dinneen@vuw.ac.nz \\ R. Tinati \\ Microsoft Corp \\ E-mail: ratinati@microsoft.com
}


Keywords information - philosophy · temporal data mining · bursts · information dynamics · socio-technical systems - information theory · information cascades · complexity science · network science · epistemology · knowledge $\cdot$ truth $\cdot$ time

\section{Introduction}

The World Wide Web (for short the Web) is the largest socio-technical system in existence, a system in which very large numbers of social agents and technical components act and interact. Despite the Web's apparent randomness and unpredictability, graphing its hyperlinks allowed the detection of some universal properties (e.g. the heavy-tailed distribution of hyperlinks between Web pages) that once gave a sufficient answer to the question of what its macroscopic structure may look like, to understand the role more or less connected (or even disconnected) parts of the Web play for the retrieval and ranking of content (Brin and Page, 1998; Broder et al., 2000), but also to learn about the human behaviour that created this structure (e.g. through preferential attachment processes) (Barabási et al., 2000; Adamic and Huberman, 2000).

While publishing HTML Web pages in the early days of the Web (i.e., Web 1.0) was a task few people were doing regularly, today more people than ever before are publishing content on the Web: from short messages on social media platforms, to content in online communities and fora, to blog posts and Wiki articles. This growth has been supported in part by an abundance of tools that make it easier for people to create and publish content without high levels of expertise and training.

One characteristic of most of these tools is that they support new content relationships that reside in patterns within the shared content itself (e.g., categories, tags or hashtags, mentions of usernames). However, in contrast to traditional hyperlinks in HTML Web pages, those new relationships are usually only explicit within one particular system; for example, hashtags within a microblogging platform are only for internal uses on a platform and do not link to the content (e.g. the same hashtags) on other platforms, turning the Web into a series of walled gardens.

A second characteristic of such tools is that they usually produce content that is explicitly time-stamped. While the Web has always been a temporallyordered artefact in the sense that all its content has been published at a particular point in time (Van de Sompel et al., 2009, 2013), contemporary tools have increased the salience of temporal relationships for understanding the associations between pieces of content.

In this article we argue on principled and practical grounds that increased temporal dynamics and implicit content relationships are challenging our understanding of the understood structure of the Web in particular and potentially of all time-evolving socio-technical systems, and that a new method for modeling that structure is needed to overcome these challenges. Our argumentation employs a di- 
alectic of literature on the philosophy of truth and science as well as analytical methods for the study of information diffusion, Web graphs and social networks in order to make a more general case for changing the current view to the actions of human collectives in the digital. We present a method for modeling information diffusion by constructing Transcendental Information Cascades (TICs), which breaks with the causality assumption implicit in many of today's analytical methods and thus allows the capture of novel dimensions of complexity of information-sharing from a macroscopic perspective. We also discuss the theoretical contributions of modeling information dynamics in this way, which holds the potential to improve our understanding of any kind of time-evolving system.

Our work adds to prior research emphasizing the role of activity and interaction sequences as the fundamental unit of analysis in socio-technical systems (Keegan et al., 2016), but further seeks to widen the scientific discourse on that matter. We acknowledge the value that lies in prior analytical methods mining causal structures and behavioral motifs from sequential data, but we also highlight that there is benefit in understanding the layers of complexity present among low-level coincidences in the various information sequences produced by socio-technical systems. What we describe here is directly related to research on socio-technical systems as investigated in computer-supported collaborative work (CSCW), Web Science, and computational social science (CSS). However, the methods we have developed and the observations we have made may also have wider implications for the study of any kind of system in which information is sequentially ordered. Examples include physiological time series such as EEG and ECG, historical text archives, and literary traditions.

The remainder of this article is structured as follows. In Section 2 we discuss why context-rich data analysis methods are limited in describing the organic nature of information in modern socio-technical systems because of their reliance on single channels of conversation, particular binary relationships, and causality between individuals' activities. In doing this we establish the notion of a complex artifact defined by relationships of meaning, which can be captured by a novel method for modeling information in Transcendental Information Cascades (TICs). After introducing TICs we describe several of their properties that can be leveraged to investigate information dynamics in sociotechnical systems in a novel way, avoiding the problems context-rich methods encounter when the aim is to get a complete picture of the macroscopic state of an entire system such as the Web. In Section 5 we describe two cases where we applied TICs to data sets obtained from the world's largest online citizen science platform Zooniverse ${ }^{1}$ and the English version of the Wikipedia ${ }^{2}$ online encyclopedia. We then discuss the findings from those two cases in relation to existing literature on information clustering, temporal data mining, computersupported cooperative work, and theories of social and information systems. In Section 6 we discuss the theoretical contributions of TICs, noting four rea-

\footnotetext{
1 https://www.zooniverse.org

2 https://en.wikipedia.org
} 
sons for thinking they may provide desirable theoretical underpinnings for the study of socio-technical systems. We conclude the article by summarizing the contributions we sought to make and outlining a few directions for a research agenda for Transcendental Information Cascades.

\section{When socially determined network models fall short}

Since the inception of the World Wide Web 29 years ago, information-sharing behavior has evolved from a relatively static picture (a rather small number of content contributors authoring individual hypertext pages with embedded hyperlinks) to one where a) content is shared at very high (and still growing) rate and b) links between content elements are increasingly implicit, as they emerge from common metadata (e.g., categories) or patterns in the actual content such as hashtags or username mentions. Many of the analytical methods that are applied to socio-technical systems (i.e., those found on the World Wide Web and most modern business information systems) are based on the assumption that there must be some retrievable snapshot of the structure underlying the behavior of the user base (e.g., a human collective using the Web) that can be used to infer causality (e.g., a social network graph). We call this assumption the causality assumption and argue that while it may hold for many particular systems, it is unlikely to hold across the heterogeneous systems that comprise the World Wide Web for example, or even all those systems in action within a single organization. Thus, the assumption poses an obstacle to macroscopic views of information and information structures.

\subsection{The problem by example}

To demonstrate the limitations of the causality assumption, let us consider the example of digitally-mediated disaster response. The earthquakes in Haiti (2010) and Nepal (2015), the political crises in Congo and Somalia, and the recent Ebola outbreak in West Africa (2014-16) are representative cases where dedicated Web applications were combined with general social media platforms to facilitate effective crisis response supported by the opportunistic gathering of crisis-related information. The information relevant to a particular crisis goes well beyond that shared by individuals to support crisis management directly; depending on the purpose of the content creator, it can be intentional (e.g., contributions via an instance of the Ushahidi tool suite) or accidental that information is relevant to relief coordination (e.g., a micropost on Twitter about one or more cancelled flights from or to a particular airport could unintentionally provide relevant information for aid workers who have to reach a crisis region from outside of that area). In such cases we see that a) crisisrelated information does not necessarily reside on a single platform and b) even when selecting a particular platform to capture crisis related information, the relevance depends on the content rather than the social networks 
that platform is supporting. As depicted in Figure 1 collective action is more salient when viewing content dynamics over time rather than by explicit social structure of a single system (Lee and Paine, 2015). It is more likely that the relevant information about an event that affects many individuals - particularly heterogeneous individuals who have only weak links with the majority of others affected - goes beyond any individual communication channel.

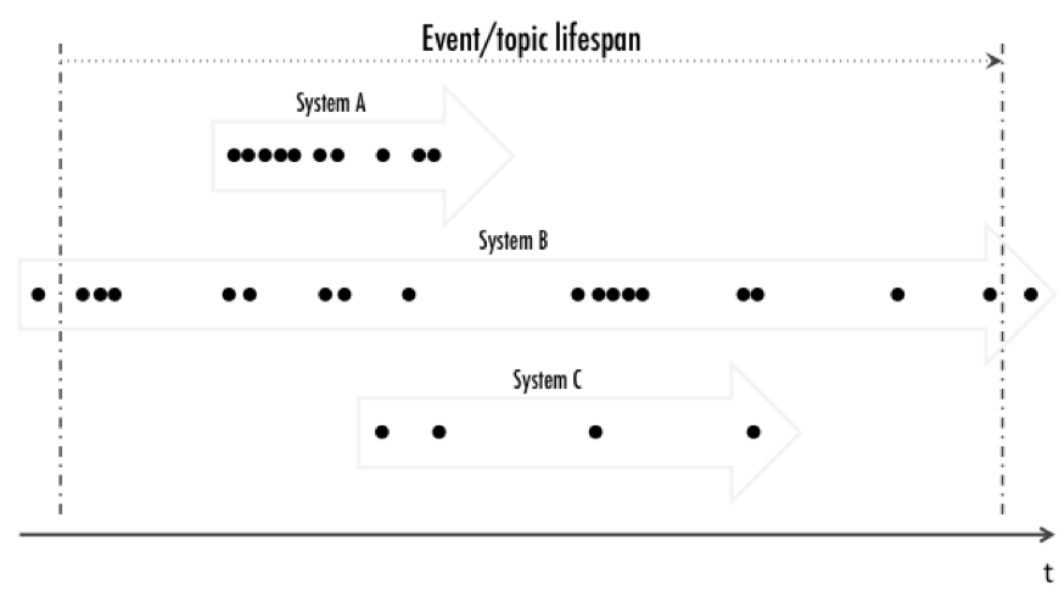

Fig. 1 Information naturally resides in an ecosystem and is emitted at varying frequencies. The accumulated information that is relevant for an event or topic forms the implicit collective action related to it.

The intuition behind our argument is that for a human conversation, or collection of conversations connected by a particular topic, the media of those conversations are of secondary importance to the participants (though of course, recalling McLuhan's famous insight, this is not to say that the medium is irrelevant to understanding the message (McLuhan and Fiore, 1967)). Human interlocutors are swayed by many factors in their choice of medium for a particular communicative act, from opportunism, to habit, to the need for security and/or anonymity, to the devices in their (and their interlocutors') possession, to requirements for synchronous discussion, to the need to reflect and to gather information before communicating, to whether the interlocutors are communicating in official or private capacities, etc. (Kraut et al., 2012). Someone who needs to communicate with someone else will use whatever is at hand, which may entail multiple venues rather than intentionally restricting the conversation to a single channel such as Twitter or email. A conversation made up of many communicative acts may therefore take place across many different media, and this will be compounded when we aggregate conversations to try to set out an inclusive information picture. Many communicative acts will take place in face-to-face speech or in other unrecorded and unrecordable ways, and so these are not going to be captured. However, a method of recording that can encompass a range of media, not restricted to any indi- 
vidual channel such as a social networking site or a microblogging site, will, all things being equal, capture more of a conversation between diverse interlocutors. To take an obvious example, a tweet from President Trump might directly cause an editorial in the New York Times, and an official response on video from the North Korean leadership on a government website. Neither of these need necessarily be immediately detectable from analysis of Twitter.

Furthermore, by examining multiple media, groups of conversations will be connectable via the exogenous events that coincidentally motivate them. This is because the occurrence of a major event, such as a crisis (e.g., an earthquake) will trigger a number of independent conversations using similar vocabulary and identifiers on similar timescales. The aggregate of these conversations may be of great importance to crisis managers or rescue workers, but not visible when focusing on individual sources of data with particular models of discussion embedded into particular information infrastructures. What is of interest, then, is the wider collective discussion, a series of conversations connected only by the basic relationships of being about the same thing and taking place during a key time period. Connecting conversations on a single channel will be facilitated by the resources of that channel, of course, but a parallel argument shows that such a narrow focus will not only capture a fraction of each conversation at best, but will also cover only a fraction of the total number of relevant conversations across the Web.

The causality assumption makes single-channel analyses useful, of course; making contextual assumptions about particulars of a conversation, for example about the connection between two communicative acts (e.g., it may be inferred from the infrastructure of a channel that $C$ ' is a reply to $C$ ), is easier if the data are taken from a single channel (e.g., only one channel's infrastructure must be described to make the inference). The opposite is true for multi-channel views, in which any two communicative acts that share a vocabulary and are closely connected in time cannot be inferred to be dialogue because they may be merely coincidental. The single channel being analysed also usually performs invaluable services to the researcher, such as certifying that the same person/device sent/received a set of communications. The analyses of multi-channel models we propose constructing are therefore necessarily low in context, at least initially, and especially so when maximally inclusive, in which cases they may consist of both genuine conversations and coincidentally related communications. However, further analyses can re-introduce contextual factors to the initial low-context model, and can then be used to pare the structure down to something more intentional. For example, after constructing the model, known factors like friendship relationships can be re-introduced (e.g., by weighting edges in the network) to allow the inference of causality. This retains the benefits of high-context analyses, when they are possible, while avoiding problems of the causality assumption, and only in such cases can we analyse a topic or conversation across channels as we have argued for. Traditional methods in this space tend to assume that the available data, for example from a social networking site, capture the complete conversation and 
its context. This is a handy assumption for researchers, but it is limited in practice and unlikely to reflect the understanding of the participants.

\subsection{The epistemological trap of system-specific analyses}

In addition to these practical problems, we note at least three different principled problems described recently in the literature that together cast further doubt upon social network analyses and the model of social contagion as means of understanding the ways in which socio-technical systems use social media to communicate, coordinate and organise. First is a doubt about what insight such traditional analyses can provide given that actors in the networks under consideration have to make their communicative decisions based on a limited view of their network. This constraint means that it is very easy to come to the wrong conclusion about the network as a whole. For instance, it is easy to construct networks where the more active or better-connected actors have non-standard opinions, so that less active actors may take their joint testimony, a local maximum only, as an indication of a consensus view (Lerman et al., 2016). This may lead them to accept a weak social justification for a belief, erroneously understanding it to be strong because each relatively inactive actor sees relatively few other inactive actors (in social networks, most people have fewer friends than their friends have) (Feld, 1991). Or genuine beliefs may be suppressed because actors erroneously think they are not shared across the network. The aggregated testimony of each actor's local neighbours will often fail to represent the network as a whole. In other words, each actor's limited view of their network can cause them to produce network artifacts that serve as red herrings, impacting any collective phenomena that network analysis is supposed to assess. Actors of high degree in the network will bias its behaviour, an effect that will be exacerbated by any algorithmic process designed to influence the actors in the network.

Second, the simplified assumption that information spreads epidemically like viruses fails to capture the typical pattern of information diffusion in social networks (Lerman, 2016). Information spreads far less widely than a viral pattern would suggest. Quite apart from the possibility that some sharing might take place via other channels (for instance, a TV report of a viral tweet might lead people to believe that all their friends have seen it, and that therefore its novelty had diminished), sharing information involves some effort to select what to share and with whom to share it. This effort is greater for well-connected agents than for poorly-connected ones; hence, unlike with a virus, well-connected agents will tend to act as brakes on the viral spread of information (bots are the exceptions that prove this rule). Meanwhile poorlyconnected ones may be more easily incorporated into an epidemic, but their meagre links mean that they won't infect many. Other cognitive heuristics to do with the way information is served up by a site mean that sharing is far less likely to occur as information becomes less recent, increasing the tendency of information epidemics to die out. In fact, this tendency for networks 
to dampen the spread of information could be helpful for communities wishing to converge on correct hypotheses, by preventing consensus being reached prematurely (Zollman, 2007; Smart, 2017).

The first two problems discussed are caused by the properties of networks and limitations of traditional analyses. The third problem is not such a mismatch, but rather a bias in the study of such systems; namely, "our selective observation of successful [cases] provides us with a false narrative of [the] underlying causes" (Cebrian et al., 2016). Cebrian et al. argue that social change is rarely effected by the rapid, bursty spread of social information, because the richer connections between the information and the potential for collective action are usually absent. Change requires a long-term commitment to overcoming opposition, as well as effective coordination; incentives to do more than simply retweet must align with the ideas and culture of the actors in the network. Social media, however, are designed for virality, not engagement or recruitment, and so scientists, as they focus on the abundant data about information diffusion, are restricted in their search to phenomena which are at best only tangentially related to important causes of behaviour. To be sure, information diffusion is a real effect, of great interest, for example, to advertisers and influencers, but it is only part of the story of opinion change, blind to other salient factors such as incentives, framing, reflection and debate (Cebrian et al., 2016).

One way of seeing this issue is to argue that a causal narrative of the spread of ideas has to goes beyond the epistemology of information diffusion, requiring discussion of practical reasoning and planning, additionally to disseminating ideas. An alternative claim is that data about which agents disseminate which ideas tells no causal story at all. Data analysis methods that have a particular social abstraction built into the model (e.g. a social network representation) impose one very specific view of information propagation. This view is on one hand obscured formally by properties of the mathematical construct of networks (Lee et al., 2017), which can skew the perception of the variety of collectives of doxastic agents (Pettit, 2006, 2010). On the other hand it reduces the sociality of justification conditions down to a situation that - at best is based on goal-instrumental social action, leaving out values, affections and cultural tradition from the full spectrum of Weber's non-positivist construction (Weber, 1978).

These three issues, that agents have (i) limited, local and often misleading views of their networks, (ii) cognitive limitations that dampen viral information propagation, and (iii) a more sophisticated relationship with the information than is captured by the data, together reveal an epistemological trap, wherein an analyst cannot know, for any particular informational pattern passing or being passed through a socio-technical system, whether it is any more than suppositio materialis (Tarski, 1944). In other words, the shared meaning and significance of a term used by a collective of individuals (on Twitter, for example) will be impossible for the analyst to access. The relationships manifested in the data about a diffusion network, do not necessarily have any defining or designating reference to the knowledge object itself or any inten- 
sional semantics at a macroscopic scale (Whitehead and Russell, 1912), and thus are inscrutable. We conclude from these problems that traditional analytical methods relying on system-specific digital structures (e.g., social network analyses) are in principle precluded from verifying they have accurately represented the socio-technical systems they examine. While such methods function sufficiently well in some applied contexts (e.g., for predicting user behavior in online advertisements), where suitability has a straightforward metric (e.g. by the advertiser making a profit), these limitations entail that they do not, and cannot, satisfy traditional notions of truth (Popper et al., 1972), a necessary goal for scientific work. The epistemological trap is sprung because the peculiar properties of social networks undermine the very assumptions made by contagion theories; the complexity of the network considered as a system or as a collective precludes the proposed information propagation mechanism from being the full epistemological story.

One hopeful question might then be: is there an analytical method for capturing the structure and dynamics of a socio-technical system that is resilient against these aforementioned issues and therefore evades the resulting limitations?

\subsection{Acknowledging complexity beyond pure reason: an entangled Web?}

As discussed above, various problems are entailed for traditional methods for analysing socio-technical systems. Are these problems theoretical or merely technical? Perhaps they are both: performing such analyses requires assuming causality (i.e., the causality assumption is part of the definition of such analyses), making it a principled problem, and the examples given above demonstrate that this results in practical problems (i.e., necessary limitations to the feature space across multiple systems as well as the scope, veracity, and conclusions of the analyses).

Given these problems, our aim in developing a desirable analytic method is to consider how we can understand evolving information in abstraction from both the social networks through which they flow and the system-specific digital traces of the social context (i.e., acausally). As outlined before, such consideration of socio-technical systems implies that system-specific data is incomplete to describe the macroscopic state of the space of all relevant information. Unconventional or hidden relationships between information, which would usually appear as noise relative to the explicit social relationships between the originators of the information, may be very influential despite, or independently of, the social structures created and curated by networking systems. Hence, we suggest separating the social context from the technological substrate to understand the Web's contribution qua abstract information space to the evolution of information. Whereas research on collective intelligence and human computation typically focuses on groups working explicitly or implicitly together towards a particular outcome and the coordination to optimize this (Malone et al., 2009; Woolley et al., 2010; Quinn and Bederson, 
2011), in this research the goal is to expose the resources produced by accumulated human activities on the Web while minimizing, entirely if possible, the presuppositions about causality or the communities and systems (i.e., single channels) in which they take part.

Towards this goal, an analytical method has been proposed in (LuczakRoesch et al., 2015b) and (Luczak-Roesch et al., 2015c). This method produces Transcendental Information Cascades, directed networks, formalised below, consisting of information recurrences characterized solely by time and inherent properties of pairs of content resources. The cascades are referred to as transcendental in Kant's sense of the word, namely, attempting to understand the conditions of knowledge itself (Kant, 1934). This means that not all such networks represent underlying purposeful activity; recurrence may simply be coincidence, but they do present a distinct set of properties of the macroscopic informational state of any socio-technical system like the Web. The relationships within and between such networks will reveal linking structures hidden from the system-specific point of view as well as temporal dependencies, describing a kind of entanglement between content resources.

\section{The Transcendental Information Cascade formalism}

We will now turn to the technicalities of Transcendental Information Cascades (Luczak-Roesch et al., 2015b,c) in order to demonstrate that there is already analytical capacity to capture the organic informational state of socially constructed information in systems, including those within other systems and across multiple channels. This shall demonstrate what tools or techniques may help understand this entangled Web independently of curated networks, and provide a reflection on two cases in which Transcendental Information Cascades are key to uncover otherwise hidden relationships between Web resources.

A Transcendental Information Cascade is defined as a directed network that is constructed from a sequence of resources (e.g., emails, blog posts, microposts, online forum entries, pages of a book, shared photos, time window snapshots of a continuous signal). Resources are ordered within the sequence (by the time of their occurrence from the oldest to most recent). Nodes in the network are those resources from this set that contain one or multiple cascade identifiers. A cascade identifier is any unique informational token that can be isolated by applying some information extraction algorithm to the original content of the elements of the sequence (e.g., natural language processing to identify unique words, phrases or entities in texts, or the color spectrum of specific areas in images). An edge exists between any two nodes that share a unique subset of all the cascade identifiers found in a sequence (e.g., two posts share some number of phrases, two images feature the same color spectrum in the same area), but only if no cascade identifier of this subset occurs in any element of the sequence between the two linked elements (i.e., assuming ordered elements A, B, and C, hashtags shared between A and C produce an 
A-C edge only when they are not also shared by B). Figure 2 shows a generalized example of this process, and examples with more detailed visualizations are provided below.

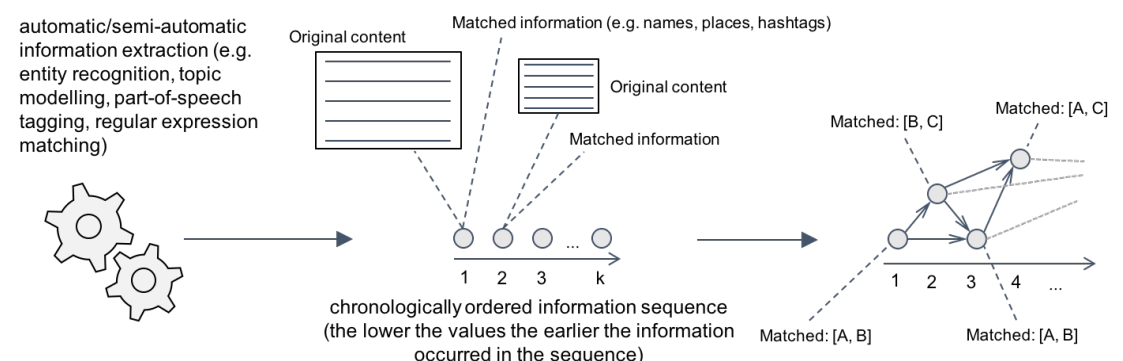
occurred in the sequence)

Fig. 2 Example of the method to construct Transcendental Information Cascades from an input sequence. For the sake of simplicity and illustrative purposes we assume that the output network was constructed by applying basic text pattern matching (in particular matching all capital letters) to textual content and that the text matched for the first four resources in the sequence was ['A','B'], ['B','C'], ['A','B'], and ['A','C'].

Formally a transcendental cascade is a tuple $T C$ that comprises a set of nodes $V$, a set of edges $E$, a set of resources $R$ and a set of matching functions $F$.

$$
T C=(V, E, R, F)
$$

Resources $R$ are defined by a unique identifier $u_{i}$, an ordering index $t_{i}$ (e.g., the time when a resource was shared), and their content $c_{i}$.

$$
R=\left\{r_{1}, r_{2}, \ldots, r_{m}\right\}, r_{i}=\left(u_{i}, t_{i}, c_{i}\right), m, i \in \mathbb{N}, i \leq m
$$

Complementary to resources exists the set of matching functions $F$.

$$
F=\left\{f_{1}, f_{2}, \ldots, f_{n}\right\}, n \in \mathbb{N}
$$

A matching function can be any algorithm that is suited to extract information from $c_{i}$ of resources $r_{i} \in R$. We define a matching function $f_{k} \in F, k \in \mathbb{N}, k \leq n$ as:

$$
f_{k}\left(c_{i}\right)=\left\{\begin{array}{l}
\left\{i_{1}, i_{2}, \ldots, i_{x}\right\} \text { if } f_{k} \text { matches patterns }\left\{i_{1}, i_{2}, \ldots, i_{x}\right\} \text { in } c_{i}, x \in \mathbb{N} \\
\emptyset \text { otherwise }
\end{array}\right.
$$

We derive a set of nodes $V$ with one corresponding node for each resource with a non-empty set of cascade identifiers $I_{i}$. Each node $v_{y} \in V$ then is described by a unique identifier $u_{y}$, the ordering index $t_{y}$, and a set of cascade identifiers 
$I_{y}$. And each set of cascade identifiers $I_{i}$ is given by the union of the results of all matching functions in $F$ applied to $c_{i}$.

$$
\begin{array}{r}
V=\left\{v_{1}, v_{2}, \ldots, v_{p}\right\}, v_{y}=\left(u_{y}, t_{y}, I_{y}\right), p, y \in \mathbb{N}, y \leq p \\
I_{i}=\left\{i_{1}, i_{2}, \ldots, i_{o}\right\}=f_{1}\left(c_{i}\right) \cup f_{2}\left(c_{i}\right) \cup \ldots \cup f_{n}\left(c_{i}\right) \\
o=\sum_{x=0}^{n}\left|f_{x}\left(c_{i}\right)\right| \\
\Rightarrow \forall i_{j} \in I_{i} \exists f_{k}\left(c_{i}\right) \rightarrow i_{j} \in f_{k}\left(c_{i}\right), j \leq o
\end{array}
$$

Edges $e_{z}$ are directed from the source node identified by $u_{a}$ to a target node identified by $u_{b}$. They exist between any two nodes $v_{a}, v_{b} \in V$ that have a common identifier subset $\Lambda_{z}$ so that $\Lambda_{z} \in I_{a}$ and $\Lambda_{z} \in I_{b}$ and furthermore no identifier has been matched in any other resource with an ordering index between $t_{a}$ and $t_{b}$.

$$
E=\left\{e_{1}, e_{2}, \ldots, e_{q}\right\}, e_{z}=\left(u_{a}, u_{b}, \Lambda_{z}\right), q, z \in \mathbb{N}, z \leq q
$$

$$
\begin{aligned}
\Lambda_{z} & =\left\{i_{r} \mid\right. \\
i_{r} \in I_{a} \wedge i_{r} \in I_{b}, \forall i_{r} \rightarrow V^{\prime}=\left\{v_{c} \mid v_{c}\right. & \left.=\left(u_{c}, t_{c}, I_{c}\right), i_{r} \in I_{c} \wedge t_{a}<t_{c}<t_{b}\right\}=\emptyset,
\end{aligned}
$$

$$
\left.v_{c} \in V, r \in \mathbb{N}, r \leq\left|I_{b}\right|\right\}
$$

The constructed networks of information token recurrence are context-free in the sense that no global feature set or pre-existing structure is exploited for their generation, and no assumptions are made about the social networking architecture used. Edges result only from the comparison of pairs of resources. That does not mean (a) that no context exists, (b) that it is unimportant, or (c) that it should not be taken into account in the investigation of the cascade, only that we need to construct the cascade as an antecedent step, rather than a resulting one, because the structures we investigate will be biased if we weave assumptions about their context into their construction. By way of analogy, we might say: a forensic scientist will gather all evidence from a crime scene before formulating hypotheses about which evidence is relevant to the crime, as she should not begin with the assumption that Miss Scarlett did it with the lead piping, and then only collect the evidence that has a bearing on that narrower question. The wider set of evidence, lacking context, may at first appear incoherent, contradictory and coincidental, but once it is constructed it can be honed down with the reintroduction of context (e.g., evidence about someone with a watertight alibi can be discounted) to produce the specific narrative of the relevant explanans. Or, as Hercule Poirot said in Agatha Christie's novel The Clocks, "But, my friend, at present you have presented me only with a pattern. There are many more things to find out." For Transcendental Information Cascades, rich context can be added after the 
cascade construction, for example by weighting edges or nodes and modifying node labels.

The process described here yields different structures depending on both the data at hand and the information extraction algorithms applied, which serve to generate the particular cascade identifiers (i.e., identify information in the data set). Algorithms can be selected opportunistically, depending on (1) what is possibly significant, and (2) what structures are unlikely to be uncovered by more conventional methods; one could imagine searching for cascade identifiers within or among hashtags, URIs, quotes, topics, keywords, images, or even semantics and sentiments. Where appearances of information seem to co-occur serendipitously, we can focus our further investigation. Even though edges are only created between directly consecutive content elements that share an identifying pattern, implicitly any resource is in interaction - or entangled - with any other resource in the connected cascade it belongs to.

\section{Studying socio-technical systems using Transcendental Information Cascades}

We anticipate some general scenarios in studying socio-technical systems that would benefit from the application of Transcendental Information Cascades: the use of a single cascade to study a process; the use of multiple cascades to understand the significance of different types of information; and a framework to tie multiple cascades together into a coherent overall picture. We discuss these in turn.

\subsection{Single cascades as a process: assessing intra-cascade properties}

The nature of Transcendental Information Cascades - that they are directed networks preserving a concise set of informational patterns for each node - allows well-established quantitative methods to be used to capture structural as well as informational properties of socially constructed information traces. The benefit of this, even with only a single cascade, is that fundamental low-level analytical methods can be used, so that the system-specific context inherent to the analysis (e.g., case-specific feature sets) is reduced, allowing for unbiased discovery of significant patterns across (a cross-section of) socio-technical systems, rather than within (and therefore illicitly assuming the centrality of) particular restricted, well-behaved, and well-understood milieu. This allows determining where significant bursts of structural and/or informational patterns first appear or fade away, indicating the emergence or shift of an underlying exogenous phenomenon and providing a trigger for sampling a particular subset from the overall content (i.e., element sequence in a particular period of time that features a burst) for a detailed and further context-driven inspection. 
4.2 Multiple cascades as a signpost of diversity: assessing inter-cascade properties

Given the intra-cascade properties identified above, can we expand an analysis to inter-cascade properties? In particular, given that different configurations of information extraction algorithms applied to the same sequence of content elements will result in differing cascade networks, one might reasonably ask: which of the resulting networks is the most appropriate representation of some underlying exogenous event or activity? Or phrased differently, one might ask: what is the appropriate information extraction algorithm configuration and process to best represent the underlying accumulated activities of human participants of the system?

An answer to this question would provide the basis for devising an adaptive approach to cascade construction. To start, entropy measures reflecting the distribution of cascade identifiers could be used to determine which matched informational patterns are associated with a certain degree of randomness. This then allows refinement of the information extraction algorithms by excluding certain patterns from consideration (e.g., specific words or hashtags used for spam on social media which tend to tie together information randomly, and thus spuriously, rather than reflecting populations' priorities). Furthermore, bursty periods of different cascade networks can overlap (or be completely disjoint), indicating a relationship between (independence of) the extracted cascade identifiers. Where there is a relationship, we can then concatenate selected information extraction algorithms to construct another alternative cascade network.

\subsection{Cohering multiple cascades: information in a multi-dimensional space}

Detecting bursts of activity is a suitable means to infer exogenous events underlying socio-technical systems but it is typically focused on individual information streams (e.g., the recurrence of individual words over time without regarding their co-occurrence with other words) (Kleinberg, 2003; Barabasi, 2005). If we model cascades of information recurrence to describe the global interconnected informational state in a socio-technical system, we can represent information in a multi-dimensional space as shown in Figure 3 so that we can see bursts occurring along different axes.

Preserving the context-free nature of the approach, three dimensions may be the natural base for this representation: (1) time (or more generally, order in the set); (2) an index of all unique cascade identifier sets extracted from data (reflecting the chronological order in which identifier sets are found); (3) an index for each unique identifier set which is incremented with each occurrence of the respective set over time. Adding context allows us to scale the number of dimensions variably (e.g., adding further dimensions for the system in which particular information occurred or the human individual who shared it). It may be, for example, that we would want to include a geographical dimension, 


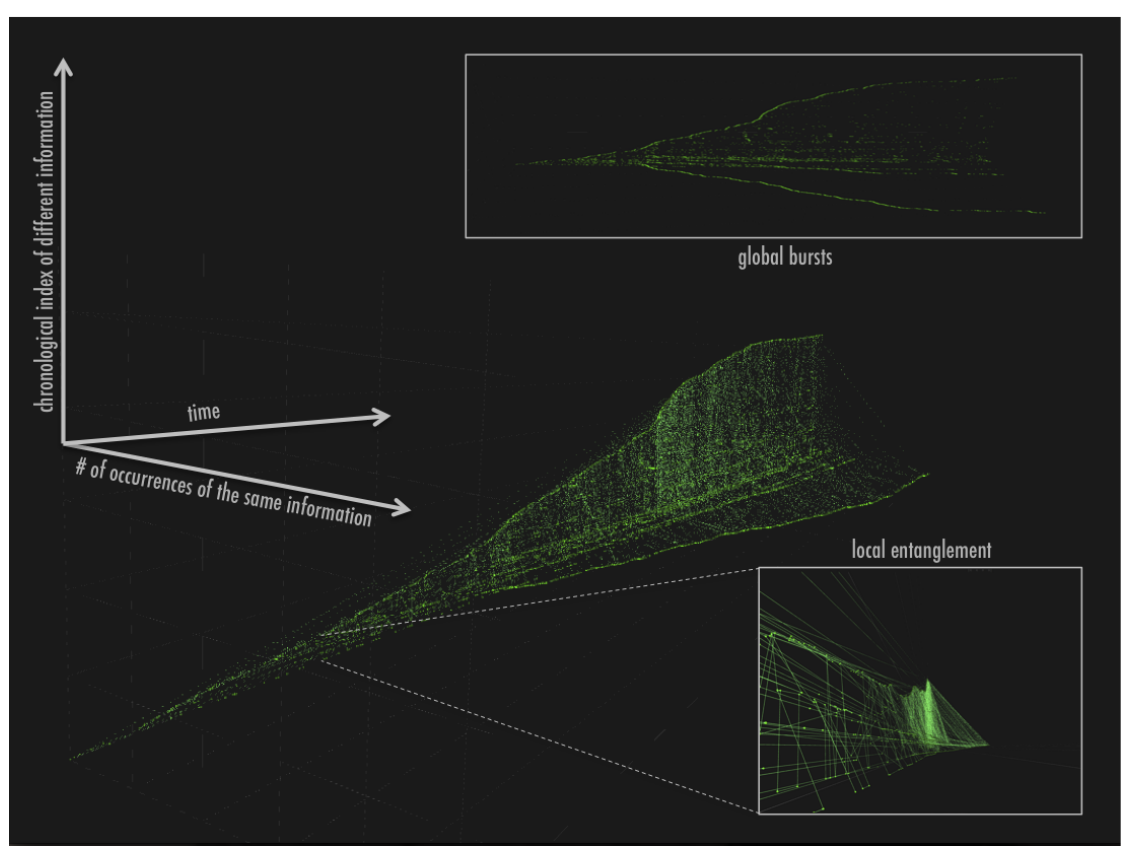

Fig. 3 Transcendental Information Cascades represented in a three dimensional space; (x) time; (y) information diversity as the chronological order in which unique identifier sets are found; (z) information specificity as the index for each unique identifier set.

because we are interested in the specific viewpoints of the heterogeneous set of actors able to influence a situation (Cebrian et al., 2016); recall the events of the so-called 'Twitter revolution' in Iran in 2009, when thanks to overreliance on the use of data from a single channel, Twitter the prospects of the revolution's success were dramatically over-estimated as most relevant Twitter traffic turned out to be supportive tweets from the US and the UK, and, as (Honari, 2015) put it, 'various areas of interest to Iranian users have been neglected or ignored' in the literature.

This projection into a three-dimensional space allows the identification of (a) periods when new unique identifier sets are created at high frequency and (b) periods when particular identifier sets burst. While this space seems to naturally diverge over time from a macroscopic viewpoint, adding the cascade links to the visualization reveals ties across individual information streams allowing the tracing of time-persistent dependencies that would be otherwise hidden.

\section{Applications of Transcendental Information Cascades}

The motivating use case described earlier was the study of digital disaster response as a collective, opportunistic and improvisatory phenomenon, but 
there are many cases where a macroscopic view of the accumulated information sharing has more value than the architecturally amplified activities of individuals in a specific socio-technical system. Following guidelines for casebased research (Benbasat et al., 1987; Eisenhardt, 1989), we investigated the application of Transcendental Information Cascades to understanding realworld cases dependent on cross-channel communication: online citizen science and editing activities in Wikipedia.

\subsection{Citizen science: coordination by content}

Online citizen science is a blueprint of the trending hybrid computing approach, coupling state-of-the-art artificial intelligence with human computation, to enable interested people to tackle problems in scientific research that are impossible to solve in a purely computational fashion (Raddick et al., 2009). The Zooniverse, for example, is the world's largest multi-project citizen science platform, with over 1.3 million volunteers contributing to projects from various domains such as astrophysics, biology or digital humanities among others. The platform gained popularity as the source of numerous citizen-led discoveries made after participants had branched out beyond the immediate system-generated constraints, discussing outliers and making other remarkable serendipitous observations while performing the crowdsourcing task (Tinati et al., 2015b). Information sharing on those platforms often evolves to become domain-specific and goal-oriented. Hence, supporting this domain-specific information sharing around the objects examined as part of the crowdsourcing task has become part of the core of many citizen science systems. However, from the point of view of research methods in information dynamics, these systems are very peculiar with respect to the online communities they form. They typically do not feature explicit social networks and the community structures that emerge implicitly are highly fluid and dependent on many aspects of context (Luczak-Roesch et al., 2014).

Transcendental Information Cascades were applied to a dataset representing content sharing on the Planet Hunters project hosted on the Zooniverse (Luczak-Roesch et al., 2015b,c). Four different information extraction algorithms based on string matching using regular expressions were tested on this dataset in order to construct alternative cascade networks: (1) hashtags; (2) matching of content that refers to specific object identifiers related to the images shown in Planet Hunters; (3) matching of identifiers used by the Planet Hunters community to refer to objects in external astrophysics databases; (4) URIs. The studies of the resulting cascade networks as well as some of their network and information theoretic properties revealed that only the information extraction algorithms 2 and 3 were suited to be combined without further adaptation (see Figure 4). The cascades constructed by applying these methods naturally showed patterns of disjoint local phenomena, which were correlated in time. Meanwhile, cascades based on hashtags tended to be either single identifier cascades or consist of multiple roots that merged and diverged 
to form a single massive connected component from which little useful information could be extracted. URI-based cascade networks tended to feature a significant fraction of independent cascades in which one particular identifier set recurred repeatedly. Hence hashtag and URI cascades would need to be refined first, until the intra-cascade properties indicated the same distinctiveness as the other two approaches. Note that, in this case at least, the identifiers that were already built into the system were less insightful compared to expressions that evolved within the community (e.g. KID identifiers) and consistent with our argument to move beyond system-specific features to uncover interesting relationships.

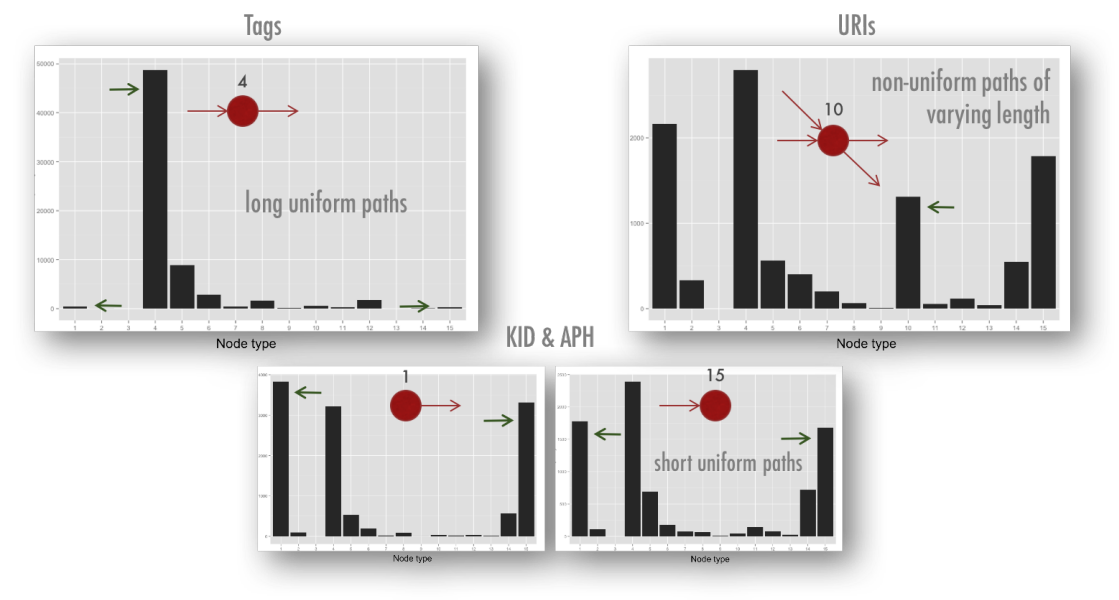

Fig. 4 The analysis of single node motifs shows the specific characteristic of the hashtag cascades (Tags). These feature a proportionally low number of network roots and stubs, which relates to many long uniform paths of hashtag recurrence that are not connected to any other matched hashtags. It is also worth highlighting the specific role of node type 10 in the URI cascades; this node type indicates that a unique matched set of URIs recurred frequently together with intermittent periods where subsets of those matched URIs occur independently. This figure was adapted from (Luczak-Roesch et al., 2015c).

5.2 Wikipedia edits: a source of temporal relationships

Wikipedia represents a network of human-curated, moderated, and maintained articles, which over time have come to comprise the largest encyclopedia in existence. The variety of social processes in Wikipedia from managing vandalism, to ensuring quality and consistency in the knowledge base, and even to detecting gender imbalances allows us to consider it as more than just a network of explicitly linked articles. Implicit structures emerge from coordinated or sometimes just accumulated activities of volunteers, but can become 
explicit if the community approves them as useful, as exemplified by WikiProjects (Tinati et al., 2015a; Tinati and Luczak-Roesch, 2017), an effort to form sub-communities in order to increase the quality of domain-specific article sets. For Wikipedia, a core challenge is to discover and in certain situations support such emergent phenomena effectively within the vast amount of user and machine-generated data. Every second, hundreds of articles are created or revised, edits are overwritten or reverted, abuses are reported, and discussions take place. This stream of activities represents the digital traces of collective human action, and to that end, studying these streams reveals temporal relationships between articles that would otherwise remain hidden and promises to provide insight into the underlying social activities of such a system from a novel angle.

As an example of the potential for progression from context-free cascade construction to context enrichment for interpretation and sense making, we applied a string matching function to the text associated with each Wikipedia revision entry. The matching function uses a regular expression to identify trigram noun phrases to match entities like 'The White House', 'Barack Hussein Obama' or 'Empire State Building' for example. In this situation Transcendental Information Cascades form a network of article edits, linked together by the shared trigrams found within the edit revision text. By enriching the article edits with contextual knowledge about article categories from DBpedia (http://dbpedia.org) it was possible to find that this cascade network represents meaningful article relationships not available within the explicit network of linked Wikipedia articles (Tinati et al., 2016).

Further analysis of the informational and structural properties as well as the general burstiness characteristics (see Figure 5 of the constructed cascades showed that they reflect both external events and phenomena inherent to the system. In particular, a burst of activity was observed featuring a series of edits made within a short duration of time beginning with identifiers found in edits on the article about Edward Snowden. The cascade then branched out to span across many other articles incorporating various identifiers related to Edward Snowden's life. A detailed inspection of the time frame when the cascade emerged showed that it coincided with a presentation given by him at the SXSW conference. In other words, a relationship between an external phenomenon and a short, bursty cascade of edits within Wikipedia, which would not have been available to a more contextualized investigation, was uncovered using the method.

In similar vein, we were also able to observe more local phenomena, such as a pathway found around the identifier: 'U.S. District Court'. This cascade extended over a longer period of time, linking articles and identifiers related to same-sex marriage in the United States, which led to an editing debate within the Wikipedia community around articles featuring lists of U.S. state laws on same-sex unions. Here, in contrast to the cascade from Snowden's talk, we were able to observe the frequent recurrence of articles within a single pathway indicating back-and-forth editing activity potentially even an edit war between Wikipedia editors. Any method that focuses either on individual users' 


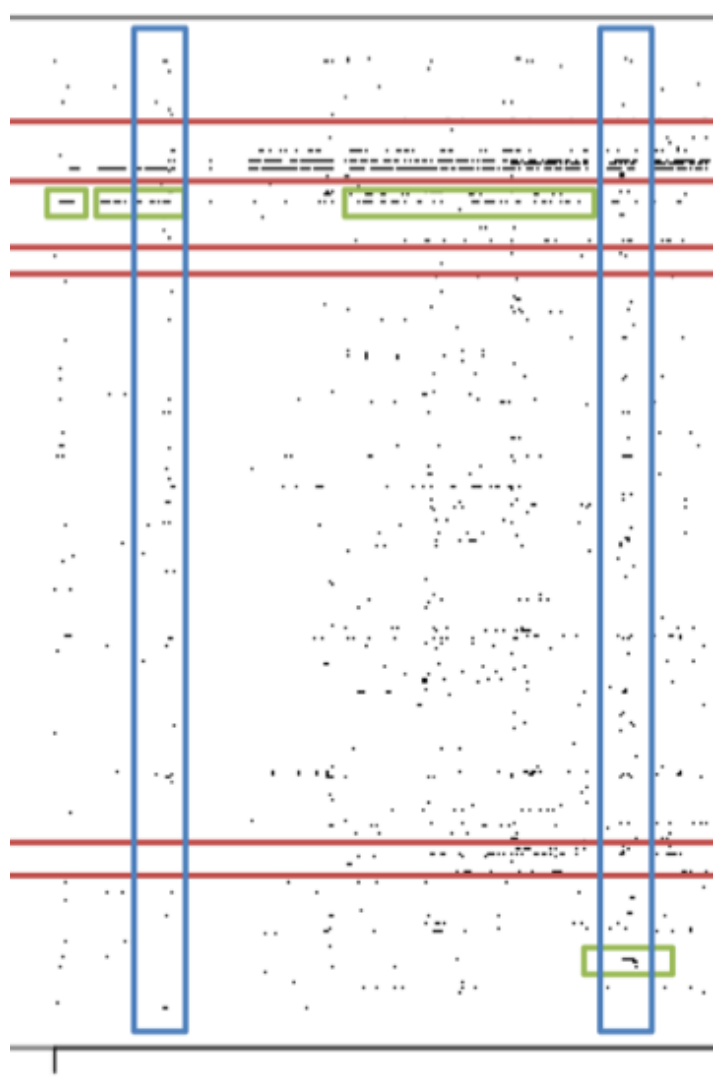

Jan

Fig. 5 Transcendental Information Cascades enabled a view that allows for differentiating between local bursts of individual trigrams (marked green), trigrams that feature continuously high editing activity as combined sets (marked red), and global bursts of activity that range across all matched trigrams (marked blue). This figure was adapted from (Tinati et al., 2016).

contributions over time or selected articles' dynamics would have missed these article relationships by shared content that were made visible only through the application of TICs.

\section{Synthesizing the case of Transcendental Information Cascades}

The applications of Transcendental Information Cascades described above show how their construction and analysis reveal hidden patterns of coordination within a stream of activity, focusing on information token recurrence 
independently from assumptions about causality, prior structure, and connectivity. This suggests that Transcendental Information Cascades may provide a unique way of underpinning the field of socio-technical systems with a distinct information-centric theory. Here we discuss a few attributes of TICs in favour of this suggestion.

6.1 Capturing objective knowledge about the macroscopic state of a socio-technical system

A common challenge in the representation and analysis of the state of sociotechical systems is the evaluation of the fitness of certain models that are the outcome of the cognitive process of a person performing the analysis. We suggest that this cognitive embedding situates those methods in 'world 2' according to Popper (Popper et al., 1972; Popper, 2013), who differentiated physical objects and events (world 1), mental objects and cognitive processes (world 2 ), and objective knowledge (world 3). Such models, situated in world 2, are not necessarily based on the same observational setup (e.g., the study of information diffusion based on modelling information cascades in a social network compared to the study of topic outbreaks modelled as activity bursts) and consequently are incommensurable views of the real state of a socio-technical system. Transcendental Information Cascades allow for an arbitrary amount of endogenous (e.g., structural properties of a particular TIC) or exogenous (e.g., features from the systems that emitted the data sequence that was transformed into that TIC) contextual features to be consulted for their analysis. However, time is the common dimension to all higher-dimensional views of different TICs of any given data sequence, and is always embedded into the network structure. Hence, we argue that time can be regarded as the only dimension independent of any context of analysis and, in consequence, independent of any cognitive model of reality imposed by the analyst. This situates Transcendental Information Cascades in world 3 according to Popper: objective knowledge about the state of a socio-technical system that can stand independently of any antecedent assumptions about what kind of network should be found (e.g., a social network is an imposed cognitive model of an online community's structure).

TICs may have application for the detection of influence of exogenous phenomena as well as temporal contagion within socio-technical systems, underlining that these contain social groupings, susceptible to influence from the full range of social contexts and social networks in which individuals take part, not simply the specific medium, platform, or architecture from which data can be harvested. In the case of Wikipedia in particular we also see great potential to uncover the injection of biases by focusing on information tokens rather than social features. We can expect people who perform such malicious editing to try to mask their identities and not to leave a digital trace allowing them to be detected and blocked. What they cannot mask is the information they inject and the TIC method allows this information to be traced over time. 
To reiterate, none of this is meant to imply that data about, or gathered from, social networks is unimportant - far from it. But some extra input is required to understand what sort of intelligence is detectable within a sociotechnical system as a whole, independently of assumptions about the mechanisms for its acquisition and delivery (and of course this independence is earned at the cost of restricting our use of assumptions about mechanism, at least as we construct the global information space).

\subsection{Transcending different views of information}

Transcendental Information Cascades allow for the specification of a range of tokens for analysis, and thus in virtue of that flexibility they avoid principled commitments to or practical problems in using one or more of the many views of information, including both qualitative and quantitative definitions (i.e., those consisting of word-based definiens and those that comprise mathematical formalisms, respectively). For example, the cascade identifiers of a TIC are consistent with data as defined in semantic conceptions of information like that provided by Floridi (2015), with conclusions drawn from analyzing such tokens thus entailing information (e.g., information about the spread of a cascade), but TICs can also be used to track information-theoretic measures derived from Shannon's quantitative view of information (Shannon, 1949), like entropy (as in Luczak-Roesch et al., 2015). As few studies have combined such complementary views (Dinneen and Brauner, 2015), it is particularly valuable that TICs enable that combination.

Further, TICs apply particularly well to two of the four views in a taxonomy of perspectives on information presented by McKinney $\mathrm{Jr}$ and Yoos (2010): the low-level matching of patterns for cascade construction entails looking for small but meaningful units in data sequences and thus reflects the 'token view' (McKinney Jr and Yoos, 2010; Lee, 2010), and the mechanism to add relationships between 'temporally coincident' (Jung, 2010) occurrences of those tokens lets the model transcend to the higher-order 'information in the syntax view' (McKinney Jr and Yoos, 2010; Lee, 2010) as an abstract type (Dinneen and Brauner, 2017).

Compatibility with (or trascendence to) the syntax view of information demonstrates further value to TICs as it suggests that a TIC channels and preserves information across time; specifically, it allows one to trace any captured information token back to the point in time when it was introduced into a socio-technical system and follow its path of co-occurrences with other tokens to the point when it eventually gets removed or replaced by another token. To our knowledge this is a unique feature of TICs, and implies that they have capacity to store and transfer information. Thus, TICs could be useful for distributed communities, which may have few communally created information storage facilities capable of allowing access to information in a timely manner (e.g., at the point at which it is needed). Some, but not all, information that can be found right from the beginning (e.g., when a socio-technical system 
is established) remain present in the most recent state of that system, so a body of information can evolve over time; information loss may correspond to information ceasing to be current, or alternatively a cascade might branch to create divergent cascades whose combined capacity may make up for apparent local losses.

\section{Discussion and Conclusion}

The aim of this work was to provide insight into a number of factors. First was the way in which the information flow is facilitated in socio-technical systems like the Web, abstracted away from the federation of co-created systems (and walled gardens). We argue that it is important to minimize the number of assumptions we make about the social context of information evolution not because we do not believe that social context plays a highly significant role, but rather to derive important social relationships from the information evolution, without reproducing the assumption that existing data from networking and sharing sites exhausts the relevant context (e.g. the misleading assumption that Facebook gives you the complete picture). This view provides an alternative, less powerful, less context-dependent, and potentially less deluded perspective on socio-technical systems in general and may be up for debate in the field as a kind of 'box-breaking research'(Alvesson and Sandberg, 2014), raising a whole set of new questions about how we model and study emergent socio-technical systems.

Transcendental Information Cascades may be a complement to analyses that rely on rich contextual features as well as more complex a priori modelling or clustering of information (Shahaf et al., 2013). For example, the novel view TICs provide to the organic structure of the information substrate produced by large scale human collectives allows for new experiments in machine learning and natural language processing that combine features derived from TICs with those available through the lens of social network analysis (or any other representation of a system-specific contextual structure). We suggest that the most promising value of such experiments lays in the development of data analytics that allow for detecting (and potentially even correcting) when certain contextual features begin to distort the analytical output.

Furthermore, the described case of Wikipedia demonstrates that the TIC approach may also complement methods that seek to detect vandalism or the injection of biases into online communities. Most approaches targeting this problem incorporate context features of the users contributing content, to differentiate valuable from malicious content (Potthast et al., 2008), but users with negative or destructive intentions are likely to try to hide their identity or deceive by planning their actions to show patterns that will not be identified by those methods. By focusing purely on information tokens, TICs are resilient against such deception, and may provide a crucial bird's-eye view to help identify the point when particular tokens got injected into an online community and how they propagated over time. 
Alternative techniques such as the ones proposed here are required and welcome, because examples such as information diffusion models leveraging social media user data (Cheng et al., 2014) show that sometimes the necessary contextual data for other methods is not available (or, under a different privacy regime, may not be accessible). The only general relationship presupposed by TICs is temporal precedence, and the key subjects of interest are recurrence (Eckmann et al., 1987; Webber Jr and Zbilut, 2005; Donner et al., 2011), or the return occurrence of information, and bursts (Kleinberg, 2003; Barabasi, 2005), or the multiple recurrences of some information tokens at high frequency.

The transcendental understanding of cascades, following our Kantian theme (Kant, 1934), is skeptical about apparent causes and seeks their necessary conditions, rejecting the ready-made etiology contained in social network data and focusing instead on the narrower supporting base of whatever is detectable from time order and syntactic/semantic coincidence. Our attempt to devise an informationcentric theory for socio-technical systems enables a macroscopic view of the emergent output of complex social action by studying the change of network and information theoretic properties, which suggests a link to social entropy theory (Bailey, 1990, 2006). This differentiates Transcendental Information Cascades from the system-centric perspectives commonly referred to in Social Computing and Computer-supported Cooperative Work (Grudin, 1994; Parameswaran and Whinston, 2007).

We hope to be able to understand any kind of socio-technical systems as a wider phenomenon than a siloed representation in a specific network might imply, a coherent phenomenon, a chord rather than its arpeggiated components, expressing its state at a time by quantifying the information represented (and its dynamics). This is valuable for research on Social Machines (Berners-Lee et al., 2000; Hendler and Berners-Lee, 2010), as characterized in (Smart et al., 2014) as 'Web-based socio-technical systems in which the human and technological elements play the role of participant machinery with respect to the mechanistic realization of system-level processes'. Our work contributes insight into the organic 'system-level processes', so that the computation of Social Machines becomes the output of this kind of analysis (Luczak-Roesch et al., 2015a), rather than one of the inputs, and no assumptions are made about Social Machines as marooning themselves on particular channels (on which we happen to have the data). This suggests that there exists an interesting new generic phenomenon in socio-technical systems that we call not necessarily coordinated collectives. A not necessarily coordinated collective is a group of people treated as equal contributors to an accumulated activity stream, regardless of any pre-defined real or virtual relationships between those people or their contributed content.

The models and experiments we have discussed here are of course very small steps on what will necessarily be a long journey of research, experimentation, and much more complex macroscopic and microscopic investigation. For instance, how do we determine the relationship between all possible Tran- 
scendental Information Cascades of a given information sequence; find the best partitioning of a cascade network into the minimum number of non-nested substructures to derive an aggregated state machine representation; or mine the collective intent of the people involved in the contents of particular Transcendental Information Cascades? It needs to be investigated whether this novel view allows for predictions of aspects of the underlying system that are at par or better than those of highly contextualized methods. And we need the capacity to index and search for not only documents and data as for classical Web graphs (Broder et al., 2000) but also Transcendental Information Cascades themselves, enabling us to understand how information dynamics facilitate and are facilitated by procedural knowledge. In the end, such understanding will have engineering repercussions, as we seek to create the conditions for the effective creation of knowledge.

TICs require extensive measurement and understanding, but many of the tools are readily at hand. They are a transformation of any kind of source data into the same well-defined temporal network model, which adds mature methods from network analysis to the analytical toolbox (Holme and Saramäki, 2012; Williams and Musolesi, 2016). And when TIC paths collide at a particular point in time, the tools of information theory (Shannon, 1949; Kullback, 1997) can be used to understand the properties of the collision and the nature of the resulting entanglement as entropy will increase or decrease. Finally, motifs of the network structure or the entropy over time can be aggregated into states, which have their own analytical apparatus grounded in stochastic processes (Anick et al., 1982; Parisi and Sourlas, 1982; Rabiner, 1989; Ovchinnikov, 2016) but also in nonlinear dynamic systems theory (Hohenberg and Halperin, 1977; Strogatz, 2014).

Beyond the application of Transcendental Information Cascades to sociotechnical systems we discussed here, TICs are a generic method that makes formerly hidden dimensions of any time-evolving system visible. We also explore the application of TICs to historic corpora of literature, EEG brain wave recordings, gene sequences and prime numbers, for example. The method always leads to the same kind of temporal network, independent from the type of input data and the analytical context, and time becomes the one dimension that is common to all possible views to the Transcendental Information Cascade in higher-dimensional space representations. This allows for seeing temporal dynamics that have not been accessible before in a unified way, and leads to novel questions about emergence, chaos, and randomness.

\section{Acknowledgement}

This article underwent an extensive open peer-review process, because the initial version was published as a pre-print on Peer $J^{3}$. The authors want thank

3 https://doi.org/10.7287/peerj fpreprints .2789 
all people who took the time to provide critical comments and feedback about this version or who engaged in discussions when the argument was presented at scientific events. Our work was partially supported under SOCIAM: The Theory and Practice of Social Machines, funded by the UK EPSRC under grant EP/J017728/2 and the Victoria University of Wellington Research Establishment Grant 8-1620-213486-3744.

\section{References}

Adamic, L. A. and Huberman, B. A. (2000). Power-law distribution of the world wide web. science, 287(5461):2115-2115.

Alvesson, M. and Sandberg, J. (2014). Habitat and habitus: Boxed-in versus box-breaking research. Organization Studies, 35(7):967-987.

Anick, D., Mitra, D., and Sondhi, M. M. (1982). Stochastic theory of a data-handling system with multiple sources. Bell Labs Technical Journal, 61(8):1871-1894.

Bailey, K. D. (1990). Social entropy theory. SUNY Press.

Bailey, K. D. (2006). Living systems theory and social entropy theory. Systems Research and Behavioral Science, 23(3):291-300.

Barabasi, A.-L. (2005). The origin of bursts and heavy tails in human dynamics. arXiv preprint cond-mat/0505371.

Barabási, A.-L., Albert, R., and Jeong, H. (2000). Scale-free characteristics of random networks: the topology of the world-wide web. Physica A: statistical mechanics and its applications, 281(1-4):69-77.

Benbasat, I., Goldstein, D. K., and Mead, M. (1987). The case research strategy in studies of information systems. MIS quarterly, pages 369-386.

Berners-Lee, T., Fischetti, M., and Foreword By-Dertouzos, M. L. (2000). Weaving the Web: The original design and ultimate destiny of the World Wide Web by its inventor. HarperInformation.

Brin, S. and Page, L. (1998). The anatomy of a large-scale hypertextual web search engine. Computer networks and ISDN systems, 30(1-7):107-117.

Broder, A., Kumar, R., Maghoul, F., Raghavan, P., Rajagopalan, S., Stata, R., Tomkins, A., and Wiener, J. (2000). Graph structure in the web. Computer networks, 33(1):309-320.

Cebrian, M., Rahwan, I., and Pentland, A. S. (2016). Beyond viral. Communications of the ACM, 59(4):36-39.

Cheng, J., Adamic, L., Dow, P. A., Kleinberg, J. M., and Leskovec, J. (2014). Can cascades be predicted? In Proceedings of the 23rd international conference on World wide web, pages 925-936. ACM.

Dinneen, J. D. and Brauner, C. (2015). Practical and philosophical considerations for defining information as well-formed, meaningful data in the information sciences. Library Trends, 63(3):378-400.

Dinneen, J. D. and Brauner, C. (2017). Information-not-thing: further problems with and alternatives to the belief that information is physical. In 
CAIS-ACSI '17: Proceedings of the 2017 Canadian Association for Information Science.

Donner, R. V., Small, M., Donges, J. F., Marwan, N., Zou, Y., Xiang, R., and Kurths, J. (2011). Recurrence-based time series analysis by means of complex network methods. International Journal of Bifurcation and Chaos, 21(04):1019-1046.

Eckmann, J.-P., Kamphorst, S. O., and Ruelle, D. (1987). Recurrence plots of dynamical systems. EPL (Europhysics Letters), 4(9):973.

Eisenhardt, K. M. (1989). Building theories from case study research. Academy of management review, 14(4):532-550.

Feld, S. L. (1991). Why your friends have more friends than you do. American Journal of Sociology, 96(6):1464-1477.

Floridi, L. (2015). Semantic Conceptions of Information. The Metaphysics Research Lab, Stanford University.

Grudin, J. (1994). Computer-supported cooperative work: History and focus. Computer, 27(5):19-26.

Hendler, J. and Berners-Lee, T. (2010). From the semantic web to social machines: A research challenge for ai on the world wide web. Artificial Intelligence, 174(2):156-161.

Hohenberg, P. C. and Halperin, B. I. (1977). Theory of dynamic critical phenomena. Reviews of Modern Physics, 49(3):435.

Holme, P. and Saramäki, J. (2012). Temporal networks. Physics reports, $519(3): 97-125$.

Honari, A. (2015). Online social research in iran: A need to offer a bigger picture. CyberOrient: The Online Journal of Virtual Middle East, 9(2).

Jung, C. G. (2010). Synchronicity: An Acausal Connecting Principle.(From Vol. 8. of the Collected Works of CG Jung)(New in Paper). Princeton University Press.

Kant, I. (1934). Critique of Pure Reason. Translated by Norman Kemp Smith. London Macmillan 1934.

Keegan, B. C., Lev, S., and Arazy, O. (2016). Analyzing organizational routines in online knowledge collaborations: A case for sequence analysis in cscw. In Proceedings of the 19th ACM Conference on Computer-Supported Cooperative Work $\&$ Social Computing, pages 1065-1079. ACM.

Kleinberg, J. (2003). Bursty and hierarchical structure in streams. Data Mining and Knowledge Discovery, 7(4):373-397.

Kraut, R. E., Resnick, P., Kiesler, S., Burke, M., Chen, Y., Kittur, N., Konstan, J., Ren, Y., and Riedl, J. (2012). Building successful online communities: Evidence-based social design. Mit Press.

Kullback, S. (1997). Information theory and statistics. Courier Corporation.

Lee, A. S. (2010). Retrospect and prospect: information systems research in the last and next 25 years. Journal of Information Technology, 25(4):336348 .

Lee, C. P. and Paine, D. (2015). From the matrix to a model of coordinated action (moca): A conceptual framework of and for cscw. In Proceedings of the 18th ACM Conference on Computer Supported Cooperative Work 6 
Social Computing, pages 179-194. ACM.

Lee, E., Karimi, F., Jo, H.-H., Strohmaier, M., and Wagner, C. (2017). Homophily explains perception biases in social networks. arXiv preprint arXiv:1710.08601.

Lerman, K. (2016). Information is not a virus, and other consequences of human cognitive limits. Future Internet, 8(2):21.

Lerman, K., Yan, X., and Wu, X.-Z. (2016). The" majority illusion" in social networks. PloS one, 11(2):e0147617.

Luczak-Roesch, M., Tinati, R., O'Hara, K., and Shadbolt, N. (2015a). Sociotechnical computation. In Proceedings of the 18th ACM Conference Companion on Computer Supported Cooperative Work $\&$ Social Computing, pages 139-142. ACM.

Luczak-Roesch, M., Tinati, R., and Shadbolt, N. (2015b). When resources collide: Towards a theory of coincidence in information spaces. In Proceedings of the 24th International Conference on World Wide Web, pages 1137-1142. ACM.

Luczak-Roesch, M., Tinati, R., Simperl, E., Van Kleek, M., Shadbolt, N., and Simpson, R. J. (2014). Why won't aliens talk to us? content and community dynamics in online citizen science. In ICWSM.

Luczak-Roesch, M., Tinati, R., Van Kleek, M., and Shadbolt, N. (2015c). From coincidence to purposeful flow? properties of transcendental information cascades. In Proceedings of the 2015 IEEE/ACM International Conference on Advances in Social Networks Analysis and Mining 2015, pages 633-638. ACM.

Malone, T. W., Laubacher, R., and Dellarocas, C. (2009). Harnessing crowds: Mapping the genome of collective intelligence. Technical Report No. 473209, MIT Sloan Research Paper.

McKinney Jr, E. H. and Yoos, C. J. (2010). Information about information: A taxonomy of views. MIS quarterly, pages 329-344.

McLuhan, M. and Fiore, Q. (1967). The medium is the message. New York, 123:126-128.

Ovchinnikov, I. V. (2016). Introduction to supersymmetric theory of stochastics. Entropy, 18(4):108.

Parameswaran, M. and Whinston, A. B. (2007). Research issues in social computing. Journal of the Association for Information Systems, 8(6):336.

Parisi, G. and Sourlas, N. (1982). Supersymmetric field theories and stochastic differential equations. Nuclear Physics B, 206(2):321-332.

Pettit, P. (2006). When to defer to majority testimony-and when not. Analysis, 66(291):179-187.

Pettit, P. (2010). Groups with minds of their own. Social Epistemology: Essential Readings, page 242.

Popper, K. (2013). Knowledge and the Body-Mind Problem: In defence of interaction. Routledge.

Popper, K. R. et al. (1972). Objective knowledge: An evolutionary approach.

Potthast, M., Stein, B., and Gerling, R. (2008). Automatic vandalism detection in wikipedia. In European conference on information retrieval, pages 663- 
668. Springer.

Quinn, A. J. and Bederson, B. B. (2011). Human computation: a survey and taxonomy of a growing field. In Proceedings of the SIGCHI conference on human factors in computing systems, pages 1403-1412. ACM.

Rabiner, L. R. (1989). A tutorial on hidden markov models and selected applications in speech recognition. Proceedings of the IEEE, 77(2):257-286.

Raddick, M. J., Bracey, G., Carney, K., Gyuk, G., Borne, K., Wallin, J., Jacoby, S., and Planetarium, A. (2009). Citizen science: status and research directions for the coming decade. AGB Stars and Related Phenomenastro 2010: The Astronomy and Astrophysics Decadal Survey, 2010:46P.

Shahaf, D., Yang, J., Suen, C., Jacobs, J., Wang, H., and Leskovec, J. (2013). Information cartography: creating zoomable, large-scale maps of information. In Proceedings of the 19th ACM SIGKDD international conference on Knowledge discovery and data mining, pages 1097-1105. ACM.

Shannon, C. E. (1949). Communication theory of secrecy systems. Bell Labs Technical Journal, 28(4):656-715.

Smart, P., Simperl, E., and Shadbolt, N. (2014). A taxonomic framework for social machines. In Social Collective Intelligence, pages 51-85. Springer.

Smart, P. R. (2017). Mandevillian intelligence. Synthese, pages 1-32.

Strogatz, S. H. (2014). Nonlinear dynamics and chaos: with applications to physics, biology, chemistry, and engineering. Hachette UK.

Tarski, A. (1944). The semantic conception of truth: and the foundations of semantics. Philosophy and phenomenological research, 4(3):341-376.

Tinati, R. and Luczak-Roesch, M. (2017). Wikipedia: a complex social machine. SIGWEB Newsletter, pages 1-10.

Tinati, R., Luczak-Roesch, M., and Hall, W. (2016). Finding structure in wikipedia edit activity: An information cascade approach. In Proceedings of the 25th International Conference Companion on World Wide Web, pages 1007-1012. International World Wide Web Conferences Steering Committee.

Tinati, R., Luczak-Roesch, M., Shadbolt, N., and Hall, W. (2015a). Using wikiprojects to measure the health of wikipedia. In Proceedings of the 24th International Conference on World Wide Web, pages 369-370. ACM.

Tinati, R., Van Kleek, M., Simperl, E., Luczak-Rösch, M., Simpson, R., and Shadbolt, N. (2015b). Designing for citizen data analysis: A cross-sectional case study of a multi-domain citizen science platform. In Proceedings of the 33rd Annual ACM Conference on Human Factors in Computing Systems, pages 4069-4078. ACM.

Van de Sompel, H., Nelson, M., and Sanderson, R. (2013). HTTP framework for time-based access to resource states-Memento. No. RFC 7089, https: //www.rfc-editor.org/info/rfc7089.

Van de Sompel, H., Nelson, M. L., Sanderson, R., Balakireva, L. L., Ainsworth, S., and Shankar, H. (2009). Memento: Time travel for the web. arXiv preprint arXiv:0911.1112.

Webber Jr, C. L. and Zbilut, J. P. (2005). Recurrence quantification analysis of nonlinear dynamical systems. Tutorials in contemporary nonlinear methods for the behavioral sciences, pages 26-94. 
Weber, M. (1978). Economy and society: An outline of interpretive sociology, volume 1. Univ of California Press.

Whitehead, A. N. and Russell, B. (1912). Principia mathematica, volume 2. University Press.

Williams, M. J. and Musolesi, M. (2016). Spatio-temporal networks: reachability, centrality and robustness. Royal Society open science, 3(6):160196.

Woolley, A. W., Chabris, C. F., Pentland, A., Hashmi, N., and Malone, T. W. (2010). Evidence for a collective intelligence factor in the performance of human groups. science, 330(6004):686-688.

Zollman, K. J. (2007). The communication structure of epistemic communities. Philosophy of science, 74(5):574-587. 